\title{
Serum immuno-biomarkers in gliomas
}

\author{
Robin A. Buerki, Rimas V. Lukas \\ Department of Neurology, University of Chicago, Chicago, IL 60637, USA.
}

Correspondence to: Dr. Rimas V. Lukas, Department of Neurology, University of Chicago, 5841 S. Maryland Avenue, MC 2030, Chicago, IL 60637, USA.E-mail: rlukas@neurology.bsd.uchicago.edu

How to cite this article: Buerki RA, Lukas RV. Serum immuno-biomarkers in gliomas. Neuroimmunol Neuroinflammation 2016;3:198-200.

Article history: Received: 29-08-2016 Accepted: 31-08-2016

Gliomas comprise the majority of malignant primary brain tumors in adults and remain a devastating diagnosis..$^{[1]}$ Despite therapeutic advances, median survival in the radiation and temozolamide era for the most common and most aggressive tumor type, glioblastoma (GBM), is 14-20 months and tumor recurrence is universal. ${ }^{[2]} \mathrm{A}$ promising new direction to combat GBM is immunotherapy, which emerged from intensive research revealing that although highly immunogenic, GBM actively suppresses the host antitumoral immune response throughout a variety of mechanisms (reviewed $\mathrm{in}^{[3]}$ and ${ }^{[4]}$ ). Current therapies to abrogate tumor immunosuppression and unleash T-cell mediated killing of GBM include: inhibitors of immune-checkpoints that are exploited by GBM to enhance tumor survival (therapeutic antibodies against CTLA-4 and PD-1, which are overexpressed in tumor-infiltrating lymphocytes, and against PD-L1, which is overexpressed on GBM cells and tumorinfiltrating immune cells); vaccination strategies against single or multiple tumor-associated peptides; infusion of autologous adoptive CAR T-cells primed against GBM-specific antigens; and inhibition of the immunomodulatory indoleamine 2,3-dioxygenase (IDO) pathway, which is also overexpressed in GBM. ${ }^{[3,5]}$ Recent work by Alexiou et al. ${ }^{[6,7]}$ published in this journal contributes to the use of immune mediators as prognostic surrogates of GBM aggressiveness
Published: 26-09-2016

and survival and may be clinical indicators of which patients are likely to benefit in current and future immunotherapeutic trials.

In the first article, a prospective, single-institution, observational study of GBM patients treated with tumor resection followed by chemotherapy and radiation (under the Stupp regimen ${ }^{[8]}$ ) for up to 1 year, Alexiou et al. ${ }^{[6]}$ demonstrate a lower neutrophilto-lymphocyte ratio (NLR) to be an independent prognostic predictor of survival. Using a cutoff value of NLR $<4.7$, both overall survival (OS) and progressionfree survival (PFS) were significantly longer. This study supports other work ${ }^{[9]}$ which also revealed NLR as an independent prognostic predictor using a cut-off value of 4 , and is consistent with other studies demonstrating the prognostic value of NLR in other tumor types. Importantly, both studies examined the NLR prior to administration of corticosteroids as corticosteroid use is itself an independent negative prognostic indicator, regardless of other chemo- or radiotherapy given. ${ }^{[10,11]}$

Extensive work has explored the role of tumorinfiltrating lymphocytes (TILs) in glioma, both as markers of tumor aggressiveness and with patient survival, namely that infiltration of cytotoxic CD8+ T lymphocytes are decreased and CD4+ and CD4+/ CD25+/FoxP3+ (Treg) populations of T lymphocytes 
increased in higher grade tumors, with increased Treg infiltration indicating a worse prognosis. ${ }^{[12]}$ Furthermore, it has been shown than TILs are enriched in the mesenchymal molecular GBM subclass, ${ }^{[13]}$ which has also been correlated with improved response to immunotherapy. ${ }^{[14]}$ Han et al. ${ }^{[15]}$ describe a prospective, single-center series of GBM patients comparing intratumoral infiltration of macrophages and $T$ cells and corroborating pre-treatment NLR (using a cut-off value of 4) with survival, establishing that serum neutrophil and lymphocytes levels (and thus the NLR) reflect the presence of TILs within the tumor milieu. In another study by Berghoff et al. ${ }^{[16]}$ a single-center retrospective analysis ("Vienna cohort"), improved survival was not associated with TIL density (or PD-L1 overexpression), in contrast the other studies. Routine analysis of TILs is difficult in clinical settings, so it is crucial that the study of Alexiou et al. ${ }^{[6,7]}$ mirrors the use of serum kynurenine and tryptophan levels as another biomarker of the immunosuppressive effect of IDO catabolism in gliomas, which demonstrated prognostic significance for $O S$ in a prospective, multi-center observational trial of GBM patients. ${ }^{[17]}$ Taken together, the work of Alexiou et al., ${ }^{[6]}$ Bambury et al.., ${ }^{[9]}$ and Han et al. ${ }^{[15]}$ elaborate upon the role of TILs in GBM via an easily obtainable, non-invasive, serum biomarker proxy. These papers reinforce and expand upon the paradigm that GBM is a lymphocyte-suppressing tumor as well as that lymphocyte down-regulation is itself a marker of an aggressive tumor pathology.

The second article by Alexiou et al., ${ }^{[7]}$ also a prospective, single-institution observational study of patients undergoing surgery for intracranial tumors (not limited to GBM), revealed that lower serum IgE levels in the GBM patients were a prognostic marker associated with poorer survival and highergrade in gliomas, although the result was of marginal significance statistically. IgE levels in gliomas and meningiomas were also significantly lower than in metastatic tumors. This supports prior work on $\lg E$ and allergies in the development of malignancies. For example, in a large, multi-center, case-controlled study of self-reported medically-diagnosed allergies, there was an inverse relationship between allergies and gliomas. Furthermore, this association was incidence responsive, the greater number of medically-diagnosed allergies the lower the glioma risk. ${ }^{[18]} A$ number of previous studies also suggest that a general atopy phenotype rather than a specific allergy type/allergen is correlated with decreased glioma risk, ${ }^{[18,19]}$ which is consistent with the total serum IgE results discussed here. Thus, it is not a limitation of Alexiou's study that allergen-specific IgE antibodies were not elucidated.
GBM continues to be a catastrophic tumor, with eventual recurrence and poor survival, in no small part due to tumor-based immunosuppression. Immunotherapy is a promising addition to the current standard of care. The studies highlighted here by Alexiou et al. ${ }^{[6,7]}$ emphasize the growing role of immune cells and antibodies in the understanding of both gliomagenesis, glioma progression, and the development of aggressive and treatment-resistant tumor types. They do not identify a causal role for certain inflammatory mediators either as protective or tumor-suppressive, but rather begin to pinpoint immune phenotypes of GBM. Furthermore, they provide easily measurable biomarkers for immunosuppressive GBM phenotypes, which are prognostic for patient survival and are clearly applicable to clinical trials for novel immunotherapies. Subsequent work is needed to correlate these findings to other prognostic GBM markers or subtypes (e.g. MGMT promoter methylation, presence of IDH1 mutations, etc.) and imaging characteristics (e.g. pseudoprogression following both standard treatment and immunotherapy is thought to be an immune-mediated response ${ }^{[20]}$ ). In fact, immunotherapy-induced changes on neuroimaging have been incorporated in to the newest response assessment guidelines ("iRANO"[21]), but whether these serum biomarkers correlate remains unclear. It is equally important to identify any mechanistic role of $\operatorname{lgE}$, and also CD4+ Th2 cells, CD4+/CD25+/FoxP3+ Treg cells, or the IgE-driving cytokines IL-4 and IL-13, in the glioma milieu and the promulgation of tumor immunosuppression. Of further value would also be to expand upon the prognostic findings described here to determine if the serum NLR or IgE levels are useful to assess treatment response.

\section{Financial support and sponsorship \\ Nil.}

\section{Conflicts of interest \\ There are no conflicts of interest.}

\section{Patient consent}

No patient involved.

\section{Ethics approval}

This article does not contain any studies with human participants or animals.

\section{REFERENCES}

1. Ostrom QT, Gittleman H, Fulop J, Liu M, Blanda R, Kromer C, Wolinsky Y, Kruchko C, Barnholtz-Sloan JS. CBTRUS statistical report: primary brain and central nervous system tumors diagnosed in the United States in 2008-2012. Neuro Oncol 2015;17 Suppl 4:iv1-62.

2. Lukas RV, Mrugala MM. Pivotal therapeutic trials for infiltrating 
gliomas and how they affect clinical practice. Neuro Oncol Pract 2016; doi:10.1093/nop/npw016.

3. Binder DC, Davis AA, Wainwright DA. Immunotherapy for cancer in the central nervous system: current and future directions. Oncoimmunology 2015;5:e1082027.

4. Lukas RV, Wainwright DA, Laterra JJ. Updates from the NeuroOncology Section of the 2015 American Neurological Association Annual Meeting. Future Oncol 2016;12:143-7.

5. Zhai L, Lauing KL, Chang AL, Dey M, Qian J, Cheng Y, Lesniak MS, Wainwright DA. The role of IDO in brain tumor immunotherapy. $J$ Neurooncol 2015;123:395-403.

6. Alexiou GA, Vartholomatos E, Zagorianakou P, Voulgaris S. Prognostic significance of neutrophil-to-lymphocyte ratio in glioblastoma. Neuroimmunol Neuroinflammation 2014;1:131-4.

7. Alexiou GA, Kallinteri A, Nita E, Zagorianakou P, Levidiotou S, Voulgaris S. Serum IgE levels in patients with intracranial tumors. Neuroimmunol Neuroinflammation 2015;2:15-7.

8. Stupp R, Mason WP, van den Bent MJ, Weller M, Fisher B, Taphoorn MJ, Belanger K, Brandes AA, Marosi C, Bogdahn U, Curschmann J, Janzer RC, Ludwin SK, Gorlia T, Allgeier A, Lacombe D, Cairncross JG, Eisenhauer E, Mirimanoff RO; European Organisation for Research and Treatment of Cancer Brain Tumor and Radiotherapy Groups; National Cancer Institute of Canada Clinical Trials Group. Radiotherapy plus concomitant and adjuvant temozolomide for glioblastoma. N Engl J Med 2005;352:987-96.

9. Bambury RM, Teo MY, Power DG, Yusuf A, Murray S, Battley JE, Drake C, O'Dea P, Bermingham N, Keohane C, Grossman SA, Moylan EJ, O'Reilly S. The association of pre-treatment neutrophil to lymphocyte ratio with overall survival in patients with glioblastoma multiforme. J Neurooncol 2013;114:149-54

10. Gorlia T, van den Bent MJ, Hegi ME, Mirimanoff RO, Weller M, Cairncross JG, Eisenhauer E, Belanger K, Brandes AA, Allgeier A, Lacombe D, Stupp R. Nomograms for predicting survival of patients with newly diagnosed glioblastoma: prognostic factor analysis of EORTC and NCIC trial 26981-22981/CE.3. Lancet Oncol 2008;9:29-38

11. Gorlia T, Stupp R, Brandes AA, Rampling RR, Fumoleau P, Dittrich C, Campone MM, Twelves CC, Raymond E, Hegi ME, Lacombe D, van den Bent MJ. New prognostic factors and calculators for outcome prediction in patients with recurrent glioblastoma: a pooled analysis of EORTC Brain Tumour Group phase I and II clinical trials. Eur $J$ Cancer 2012;48:1176-84.

12. Han S, Zhang C, Li Q, Dong J, Liu Y, Huang Y, Jiang T, Wu A. Tumour-infiltrating CD4(+) and CD8(+) lymphocytes as predictors of clinical outcome in glioma. Br J Cancer 2014;110:2560-8.

13. Rutledge WC, Kong J, Gao J, Gutman DA, Cooper LA, Appin C, Park Y, Scarpace L, Mikkelsen T, Cohen ML, Aldape KD, McLendon RE, Lehman NL, Miller CR, Schniederjan MJ, Brennan CW, Saltz JH, Moreno CS, Brat DJ. Tumor-infiltrating lymphocytes in glioblastoma are associated with specific genomic alterations and related to transcriptional class. Clin Cancer Res 2013;19:4951-60.

14. Prins RM, Soto H, Konkankit V, Odesa SK, Eskin A, Yong WH, Nelson SF, Liau LM. Gene expression profile correlates with T-cell infiltration and relative survival in glioblastoma patients vaccinated with dendritic cell immunotherapy. Clin Cancer Res 2011;17:1603-15.

15. Han S, Liu Y, Li Q, Li Z, Hou H, Wu A. Pre-treatment neutrophil-tolymphocyte ratio is associated with neutrophil and T-cell infiltration and predicts clinical outcome in patients with glioblastoma. $B M C$ Cancer 2015;15:617.

16. Berghoff AS, Kiesel B, Widhalm G, Rajky O, Ricken G, Wöhrer A, Dieckmann K, Filipits M, Brandstetter A, Weller M, Kurscheid S, Hegi ME, Zielinski CC, Marosi C, Hainfellner JA, Preusser M, Wick W. Programmed death ligand 1 expression and tumor-infiltrating lymphocytes in glioblastoma. Neuro Oncol 2015;17:1064-75.

17. Zhai L, Dey M, Lauing KL, Gritsina G, Kaur R, Lukas RV, Nicholas MK, Rademaker AW, Dostal CR, McCusker RH, Raizer JJ, Parsa AT, Bloch O, Wainwright DA. The kynurenine to tryptophan ratio as a prognostic tool for glioblastoma patients enrolling in immunotherapy. J Clin Neurosci 2015;22:1964-8.

18. McCarthy BJ, Rankin K, Il'yasova D, Erdal S, Vick N, Ali-Osman F, Bigner DD, Davis F. Assessment of type of allergy and antihistamine use in the development of glioma. Cancer Epidemiol Biomarkers Prev 2011;20:370-8.

19. Wigertz A, Lönn S, Schwartzbaum J, Hall P, Auvinen A, Christensen HC, Johansen C, Klaeboe L, Salminen T, Schoemaker MJ, Swerdlow AJ, Tynes T, Feychting M. Allergic conditions and brain tumor risk. Am J Epidemiol 2007;166:941-50.

20. Huang RY, Neagu MR, Reardon DA, Wen PY. Pitfalls in the neuroimaging of glioblastoma in the era of antiangiogenic and immuno/targeted therapy - detecting illusive disease, defining response. Front Neurol 2015;6:33.

21. Okada H, Weller M, Huang R, Finocchiaro G, Gilbert MR, Wick W Ellingson BM, Hashimoto N, Pollack IF, Brandes AA, Franceschi E, Herold-Mende C, Nayak L, Panigrahy A, Pope WB, Prins R, Sampson JH, Wen PY, Reardon DA. Immunotherapy response assessment in neuro-oncology: a report of the RANO working group. Lancet Oncol 2015;16:e534-42. 